\title{
EQUATIONAL DESCRIPTION OF PSEUDOVARIETIES OF HOMOMORPHISMS *
}

\author{
MiCHAL KUNC ${ }^{1}$
}

\begin{abstract}
The notion of pseudovarieties of homomorphisms onto finite monoids was recently introduced by Straubing as an algebraic characterization for certain classes of regular languages. In this paper we provide a mechanism of equational description of these pseudovarieties based on an appropriate generalization of the notion of implicit operations. We show that the resulting metric monoids of implicit operations coincide with the standard ones, the only difference being the actual interpretation of pseudoidentities. As an example, an equational characterization of the pseudovariety corresponding to the class of regular languages in $A C^{0}$ is given.
\end{abstract}

Mathematics Subject Classification. 20M35, 68Q70.

\section{INTRODUCTION}

Starting from Schützenberger's characterization of star-free languages in [6], several results describing certain combinatorial properties of regular languages by means of their syntactic semigroups were published. Later Eilenberg [3] gave a general framework for these results by revealing the correspondence between varieties of regular languages, i.e. classes of regular languages closed under boolean operations, quotients and pre-images under homomorphisms, and pseudovarieties of finite monoids, i.e. classes of finite monoids closed under taking homomorphic images, submonoids and finite direct products.

\footnotetext{
Keywords and phrases. pseudovariety, pseudoidentity, implicit operation, variety of regular languages, syntactic homomorphism.

* Supported by the grant 201/01/0323 of the Grant Agency of the Czech Republic.

${ }^{1}$ Department of Mathematics, Masaryk University, Janáčkovo nám. 2a, 66295 Brno, Czech Republic; URL: http://www.math.muni.cz/ kunc; e-mail: kunc@math.muni.cz
}

(c) EDP Sciences 2003 
But many naturally arising classes of regular languages fail to satisfy some of the axioms of varieties, so in order to apply standard techniques of logic and algebra one needs to consider appropriate generalizations of the notion of varieties. A useful tool appears to be the notion of positive varieties introduced by Pin [4] arising by dropping the requirement on closure under complements. Recently Straubing [7] considered classes of regular languages which satisfy all axioms of varieties except they are closed under pre-images only for a certain class of homomorphisms. He suggested a generalization of pseudovarieties of finite monoids to so-called pseudovarieties of homomorphisms from free finitely generated monoids onto finite monoids and proved an Eilenberg-type correspondence for these classes. The consideration of homomorphisms instead of plain monoids allows to capture also some properties of languages whose validity depends not only on the syntactic monoid of a language but also on its syntactic homomorphism, i.e. one takes into account which elements of the monoid correspond to the letters of the alphabet. Straubing also dealt with logical characterizations of these pseudovarieties. In this paper we provide their equational counterpart using appropriate generalizations of the notions of implicit operations and pseudoidentities.

The paper is structured as follows. First we recall the definition of pseudovarieties of homomorphisms; for a more extensive description of the concept and for several examples see [7]. In Section 2 we introduce generalized implicit operations and we show that the arising metric monoids of implicit operations coincide with the standard ones; this means that one can use the standard syntax for writing generalized pseudoidentities, which differ from the standard ones just by their interpretation. In this setting the theory of implicit operations, pseudoidentities and free profinite structures can be developed analogously to the standard case. Since most of the arguments are very similar, we only justify our claim that the notion of pseudoidentities introduced in this paper is the appropriate one by proving a corresponding generalization of the fact that pseudovarieties are precisely classes of finite monoids definable by pseudoidentities (which is the monoid version of Reiterman's famous theorem [5]). This is the aim of Section 3.

In [7] Straubing described a way to produce a pseudovariety of homomorphisms from a standard pseudovariety of semigroups by considering stable subsemigroups of homomorphisms. For instance, the class of regular languages in $A C^{0}$ corresponds to a pseudovariety created in this way. In Section 4 we show how to obtain a basis of pseudoidentities for every such pseudovariety from a basis of pseudoidentities of the original pseudovariety of semigroups.

For the necessary background on the theory of pseudoidentities the reader is referred to [1].

\section{Pseudovarieties of Homomorphisms}

In this section we recall the concept of pseudovarieties of homomorphisms introduced in [7]. 
Let us consider a fixed set $\mathcal{A}$ of finite sets and refer to elements of $\mathcal{A}$ as finite alphabets. Let $\mathcal{C}$ be an arbitrary category of homomorphisms between free monoids over finite alphabets, i.e. its objects are all monoids of the form $\Sigma^{*}$, where $\Sigma$ is a finite alphabet (from $\mathcal{A})$, and for each pair of alphabets $\Sigma, \Xi$ a set $\mathcal{C}\left(\Sigma^{*}, \Xi^{*}\right)$ of monoid homomorphisms from $\Sigma^{*}$ to $\Xi^{*}$ is given in such a way that id $\Sigma_{\Sigma^{*}} \in \mathcal{C}\left(\Sigma^{*}, \Sigma^{*}\right)$ for every $\Sigma$ and if $f \in \mathcal{C}\left(\Sigma^{*}, \Xi^{*}\right)$ and $g \in \mathcal{C}\left(\Xi^{*}, \Gamma^{*}\right)$ then their composition $g f$ belongs to $\mathcal{C}\left(\Sigma^{*}, \Gamma^{*}\right)$. Throughout the paper, $\mathcal{C}$ always denotes such a category of homomorphisms and $\mathcal{H}$ denotes the largest of these categories, where $\mathcal{H}\left(\Sigma^{*}, \Xi^{*}\right)$ is the set of all homomorphisms from $\Sigma^{*}$ to $\Xi^{*}$ for every $\Sigma$ and $\Xi$.

Note 1.1. We assume that all finite alphabets together form a set in order to be able to characterize every generalized pseudovariety by a set of pseudoidentities; if we had a proper class of alphabets, there would exist generalized pseudovarieties which can be defined only using a proper class of pseudoidentities.

The following definition of $\mathcal{C}$-pseudovarieties mimics the definition of standard pseudovarieties - the class should be closed under taking factors, substructures (depending on $\mathcal{C}$ ) and finite direct products.

Let $\mathbf{M}$ be the class of all surjective homomorphisms $\varphi: \Sigma^{*} \rightarrow M$, where $\Sigma$ is a finite alphabet and $M$ is a finite monoid.

Note 1.2. We use the same notation for a mapping no matter what its codomain is since it will be always clear from the context. In particular, if we talk about a non-surjective homomorphism $\varphi: \Sigma^{*} \rightarrow M$ as an element of $\mathbf{M}$, we actually mean the corresponding surjective homomorphism from $\Sigma^{*}$ onto im $(\varphi)$.

Definition 1.3. A subclass $\mathbf{V}$ of $\mathbf{M}$ is called a $\mathcal{C}$-pseudovariety whenever it satisfies the following conditions:

(1) If $\varphi: \Sigma^{*} \rightarrow M$ belongs to $\mathbf{V}$ and $\alpha: M \rightarrow N$ is a surjective homomorphism, then $\alpha \varphi \in \mathbf{V}$.

(2) If $\varphi: \Sigma^{*} \rightarrow M$ belongs to $\mathbf{V}$ and $f \in \mathcal{C}\left(\Xi^{*}, \Sigma^{*}\right)$, then also the homomorphism $\varphi f: \Xi^{*} \rightarrow \operatorname{im}(\varphi f) \subseteq M$ is in $\mathbf{V}$.

(3) If $n$ is a non-negative integer and homomorphisms $\varphi_{i}: \Sigma^{*} \rightarrow M_{i}$ for $i=1, \ldots, n$ belong to $\mathbf{V}$, then so does the homomorphism

$$
\left(\varphi_{1}, \ldots, \varphi_{n}\right): \Sigma^{*} \rightarrow \operatorname{im}\left(\varphi_{1}, \ldots, \varphi_{n}\right) \subseteq M_{1} \times \cdots \times M_{n} .
$$

The meaning of this definition for particular values of $\mathcal{C}$ is discussed in [7]; let us only mention that $\mathcal{H}$-pseudovarieties can be viewed as standard monoid pseudovarieties, and for the category $\mathcal{C}$ containing exactly non-erasing homomorphisms, $\mathcal{C}$-pseudovarieties can be viewed as standard pseudovarieties of semigroups.

\section{IMPLICIT OPERATIONS}

The basic concept of the equational theory of pseudovarieties is the notion of implicit operations, which consist of operations of a given arity defined on every finite monoid and invariant under monoid homomorphisms. More precisely, for 
an arbitrary positive integer $k$, a $k$-ary implicit operation is a system of mappings $\tau=\left(\tau_{M}: M^{k} \rightarrow M\right)$, for all finite monoids $M$, such that for every monoid homomorphism $\alpha: M \rightarrow N$ the diagram

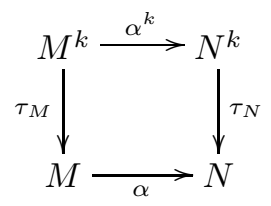

commutes, where $\alpha^{k}\left(e_{1}, \ldots, e_{k}\right)=\left(\alpha\left(e_{1}\right), \ldots, \alpha\left(e_{k}\right)\right)$ for any $e_{1}, \ldots, e_{k} \in M$. We denote the set of all $k$-ary implicit operations by $\mathcal{I}_{k}$. A prominent role among implicit operations is played by term operations; in fact, every $k$-ary implicit operation can be expressed as a limit of $k$-ary term operations.

In the case of $\mathcal{C}$-pseudovarieties the situation is similar, with only two major differences:

- An implicit $\mathcal{C}$-operation consists of partial operations whose domains are the sets of so-called $\mathcal{C}$-admissible tuples.

- Arities of implicit $\mathcal{C}$-operations are arbitrary finite alphabets; in general, implicit $\mathcal{C}$-operations for arities of the same cardinality can be different.

Let $\Gamma$ be a finite alphabet. For a homomorphism $\varphi: \Sigma^{*} \rightarrow M$ in $\mathbf{M}$ we denote by $M_{\varphi, \mathcal{C}}^{\Gamma}$ the set $\left\{\varphi \iota \mid \iota \in \mathcal{C}\left(\Gamma^{*}, \Sigma^{*}\right)\right\}$ consisting of homomorphisms from $\Gamma^{*}$ to $M$ and call its elements $\mathcal{C}$-admissible $\Gamma$-tuples for $\varphi$. Notice that if the alphabet $\Gamma=\left\{\gamma_{1}, \ldots, \gamma_{k}\right\}$ has exactly $k$ elements, then homomorphisms from $\Gamma^{*}$ to $M$ correspond to $k$-tuples of elements of $M$, so we can conceive $M_{\varphi, \mathcal{C}}^{\Gamma}$ as a subset of $M^{k}$; every $\varphi \iota \in M_{\varphi, \mathcal{C}}^{\Gamma}$ represents the $k$-tuple $\left(\varphi \iota\left(\gamma_{1}\right), \ldots, \varphi \iota\left(\gamma_{k}\right)\right) \in M^{k}$.

For two homomorphisms $\varphi: \Sigma^{*} \rightarrow M$ and $\psi: \Xi^{*} \rightarrow N$ in $\mathbf{M}$, a monoid homomorphism $\alpha: M \rightarrow N$ is termed a $\mathcal{C}$-admissible morphism from $\varphi$ to $\psi$ if there exists $f \in \mathcal{C}\left(\Sigma^{*}, \Xi^{*}\right)$ making the following diagram commute:

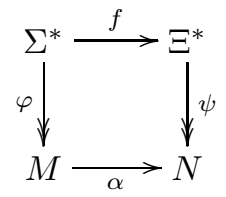

If $\alpha$ is a $\mathcal{C}$-admissible morphism from $\varphi$ to $\psi$, then the rule $\alpha^{\Gamma}(\varphi \iota)=\alpha \varphi \iota$, for each $\iota \in \mathcal{C}\left(\Gamma^{*}, \Sigma^{*}\right)$, determines a mapping from $M_{\varphi, \mathcal{C}}^{\Gamma}$ to $N_{\psi, \mathcal{C}}^{\Gamma}$. Indeed, as there exists a homomorphism $f \in \mathcal{C}\left(\Sigma^{*}, \Xi^{*}\right)$ satisfying $\alpha \varphi=\psi f$, we have $\alpha^{\Gamma}(\varphi \iota)=$ $\alpha \varphi \iota=\psi f \iota \in N_{\psi, \mathcal{C}}^{\Gamma}$ since $f \iota \in \mathcal{C}\left(\Gamma^{*}, \Xi^{*}\right)$. When we understand $M_{\varphi, \mathcal{C}}^{\Gamma}$ and $N_{\psi, \mathcal{C}}^{\Gamma}$ as subsets of $M^{k}$ and $N^{k}$ respectively, the mapping $\alpha^{\Gamma}$ is nothing but the appropriate restriction of $\alpha^{k}$. Notice that because $\mathcal{C}$ is a category, we obtain for every $\mathcal{C}$ a category whose objects are the elements of $\mathbf{M}$ and whose morphisms are the $\mathcal{C}$-admissible morphisms; for $\mathcal{C}=\mathcal{H}$ this category is equivalent to the category 
of finite monoids and for $\mathcal{C}$ consisting of all non-erasing homomorphisms, it is equivalent to the category of finite semigroups.

Now we are ready to define implicit $\mathcal{C}$-operations.

Definition 2.1. Let $\Gamma$ be an arbitrary finite alphabet. A system of mappings $\pi=\left(\pi_{\varphi}: M_{\varphi, \mathcal{C}}^{\Gamma} \rightarrow M\right)_{\varphi \in \mathbf{M}}$, for all homomorphisms $\varphi: \Sigma^{*} \rightarrow M$ in $\mathbf{M}$, is called a $\Gamma$-ary implicit $\mathcal{C}$-operation if for every $\mathcal{C}$-admissible morphism $\alpha$ from $\varphi: \Sigma^{*} \rightarrow M$ to $\psi: \Xi^{*} \rightarrow N$ the following diagram commutes:

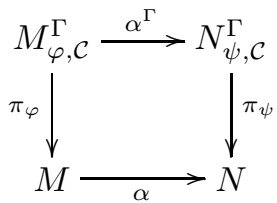

The set of all $\Gamma$-ary implicit $\mathcal{C}$-operations is denoted $\mathcal{I}_{\Gamma}^{\mathcal{C}}$; we write simply $\mathcal{I}_{\Gamma}$ instead of $\mathcal{I}_{\Gamma}^{\mathcal{H}}$.

Note 2.2. Notice how the simplification of notation introduced in Note 1.2 applies to implicit $\mathcal{C}$-operations: for a $\Gamma$-ary implicit $\mathcal{C}$-operation $\pi$ and homomorphisms $\varphi: \Sigma^{*} \rightarrow M$ and $\chi: \Gamma^{*} \rightarrow M$, in the expression $\pi_{\varphi}(\chi)$ both mappings $\varphi$ and $\chi$ are considered to have co-domain $\operatorname{im}(\varphi)$.

Next we introduce a metric on $\mathcal{I}_{\Gamma}^{\mathcal{C}}$. Consider two $\Gamma$-ary implicit $\mathcal{C}$-operations $\pi=\left(\pi_{\varphi}\right)_{\varphi \in \mathbf{M}}$ and $\rho=\left(\rho_{\varphi}\right)_{\varphi \in \mathbf{M}}$. Let

$$
r=\min \left\{|M| \mid \exists \varphi \in \mathbf{M}, \varphi: \Sigma^{*} \rightarrow M, \pi_{\varphi} \neq \rho_{\varphi}\right\},
$$

where $|M|$ denotes the cardinality of $M$, and define $\mathrm{d}(\pi, \rho)=2^{-r}$. Then clearly $\mathrm{d}(\pi, \sigma) \leq \max \{\mathrm{d}(\pi, \rho), \mathrm{d}(\rho, \sigma)\}$ for arbitrary $\pi, \rho, \sigma \in \mathcal{I}_{\Gamma}^{\mathcal{C}}$ and so $\mathrm{d}$ is an ultrametric on $\mathcal{I}_{\Gamma}^{\mathcal{C}}$.

Let us now discuss the above definitions in the case of the category $\mathcal{H}$.

Remark 2.3. Let $k$ be a positive integer and let $\Gamma=\left\{\gamma_{1}, \ldots, \gamma_{k}\right\}$ be a $k$-element alphabet. Then the set $M_{\varphi, \mathcal{H}}^{\Gamma}$ contains in fact all $k$-tuples of elements of $M$, every monoid homomorphism $\alpha$ is $\mathcal{H}$-admissible and the mapping $\alpha^{\Gamma}$ is the same as $\alpha^{k}$. Therefore the commutative diagrams defining standard implicit operations and implicit $\mathcal{H}$-operations coincide and we get a natural one-to-one correspondence between $\mathcal{I}_{k}$ and $\mathcal{I}_{\Gamma}$. More precisely, the implicit $\mathcal{H}$-operation $\pi$ corresponding to an implicit operation $\tau$ is given by the rule $\pi_{\varphi}(\varphi \iota)=\tau_{M}\left(\varphi \iota\left(\gamma_{1}\right), \ldots, \varphi \iota\left(\gamma_{k}\right)\right)$ for every $\varphi: \Sigma^{*} \rightarrow M$ in $\mathbf{M}$ and every homomorphism $\iota: \Gamma^{*} \rightarrow \Sigma^{*}$.

Moreover, if we consider the point-wise multiplication on both $\mathcal{I}_{k}$ and $\mathcal{I}_{\Gamma}$, we obtain isomorphic monoids. And because the metric just introduced on $\mathcal{I}_{\Gamma}$ also coincides with the standard metric on $\mathcal{I}_{k}$, we can reformulate well-known results about $\mathcal{I}_{k}$ using the notation of $\mathcal{I}_{\Gamma}$ as follows.

First, the metric space $\mathcal{I}_{\Gamma}$ is compact (see Prop. 3.4.6 in [1]) and the multiplication on $\mathcal{I}_{\Gamma}$ is a uniformly continuous mapping, hence $\mathcal{I}_{\Gamma}$ is a metric monoid. If 
we further consider the injective monoid homomorphism $\nu_{\Gamma}: \Gamma^{*} \rightarrow \mathcal{I}_{\Gamma}$ sending each $\Gamma$-ary monoid term $t \in \Gamma^{*}$ to the implicit $\mathcal{H}$-operation corresponding to the term operation defined by $t$, we obtain the so-called free profinite monoid over $\Gamma$. In particular, for every finite monoid $M$ (equipped with the discrete topology) and every homomorphism $\varphi: \Gamma^{*} \rightarrow M$ in $\mathbf{M}$ there exists a unique continuous homomorphism $\mu: \mathcal{I}_{\Gamma} \rightarrow M$ such that $\mu \nu_{\Gamma}=\varphi$; this homomorphism is defined by the rule $\mu(\pi)=\pi_{\varphi}(\varphi)$.

In the following proposition we show that for every category $\mathcal{C}$ the metric space $\mathcal{I}_{\Gamma}^{\mathcal{C}}$ is naturally isometric to the standard space of implicit operations. In fact, from an implicit $\mathcal{H}$-operation we obtain an implicit $\mathcal{C}$-operation by restricting to $\mathcal{C}$-admissible $\Gamma$-tuples. Conversely, in order to extend an implicit $\mathcal{C}$-operation to the whole $M_{\varphi, \mathcal{H}}^{\Gamma}$, we have to consider for each $\Gamma$-tuple of elements of $M$ the homomorphism from $\Gamma^{*}$ onto the submonoid of $M$ generated by these elements and use the fact that $\psi=\psi \operatorname{id}_{\Gamma^{*}} \in N_{\psi, \mathcal{C}}^{\Gamma}$ for every homomorphism $\psi: \Gamma^{*} \rightarrow N$ in $\mathbf{M}$.

Proposition 2.4. Let $\Gamma$ be a finite alphabet. Then the mappings $i_{\Gamma}^{\mathcal{C}}: \mathcal{I}_{\Gamma} \rightarrow \mathcal{I}_{\Gamma}^{\mathcal{C}}$ and $j_{\Gamma}^{\mathcal{C}}: \mathcal{I}_{\Gamma}^{\mathcal{C}} \rightarrow \mathcal{I}_{\Gamma}$, defined for every implicit $\mathcal{H}$-operation $\pi \in \mathcal{I}_{\Gamma}$, every implicit $\mathcal{C}$-operation $\rho \in \mathcal{I}_{\Gamma}^{\mathcal{C}}$ and for homomorphisms $\varphi: \Sigma^{*} \rightarrow M$ in $\mathbf{M}, \iota \in \mathcal{C}\left(\Gamma^{*}, \Sigma^{*}\right)$ and $\kappa \in \mathcal{H}\left(\Gamma^{*}, \Sigma^{*}\right)$ by the rules

$$
\begin{aligned}
i_{\Gamma}^{\mathcal{C}}(\pi)_{\varphi}(\varphi \iota) & =\pi_{\varphi}(\varphi \iota) \\
j_{\Gamma}^{\mathcal{C}}(\rho)_{\varphi}(\varphi \kappa) & =\rho_{\varphi \kappa}(\varphi \kappa),
\end{aligned}
$$

are mutually inverse isometries of the metric spaces $\mathcal{I}_{\Gamma}$ and $\mathcal{I}_{\Gamma}^{\mathcal{C}}$.

Proof. It is plain to verify that $i_{\Gamma}^{\mathcal{C}}(\pi)$ is indeed an implicit $\mathcal{C}$-operation. Let us prove that $j_{\Gamma}^{\mathcal{C}}(\rho)$ is an implicit $\mathcal{H}$-operation for every implicit $\mathcal{C}$-operation $\rho$. Consider any $\varphi: \Sigma^{*} \rightarrow M$ and $\psi: \Xi^{*} \rightarrow N$ in $\mathbf{M}$ and a homomorphism $\alpha: M \rightarrow N$. We have to prove $\alpha\left(j_{\Gamma}^{\mathcal{C}}(\rho)\right)_{\varphi}=j_{\Gamma}^{\mathcal{C}}(\rho)_{\psi} \alpha^{\Gamma}$. In order to do this, let $\kappa: \Gamma^{*} \rightarrow \Sigma^{*}$ be an arbitrary homomorphism. Then $\alpha\left(j_{\Gamma}^{\mathcal{C}}(\rho)_{\varphi}(\varphi \kappa)\right)=\alpha\left(\rho_{\varphi \kappa}(\varphi \kappa)\right)$ and $j_{\Gamma}^{\mathcal{C}}(\rho)_{\psi}\left(\alpha^{\Gamma}(\varphi \kappa)\right)=j_{\Gamma}^{\mathcal{C}}(\rho)_{\psi}(\alpha \varphi \kappa)=\rho_{\alpha \varphi \kappa}(\alpha \varphi \kappa)=\rho_{\alpha \varphi \kappa}\left(\left(\left.\alpha\right|_{\operatorname{im}(\varphi \kappa)}\right)^{\Gamma}(\varphi \kappa)\right)$. Since $\left.\alpha\right|_{\operatorname{im}(\varphi \kappa)}$ is a $\mathcal{C}$-admissible morphism from $\varphi \kappa$ to $\alpha \varphi \kappa$ (the identity on $\Gamma^{*}$ is a corresponding homomorphism from $\mathcal{C}$ ), the diagram

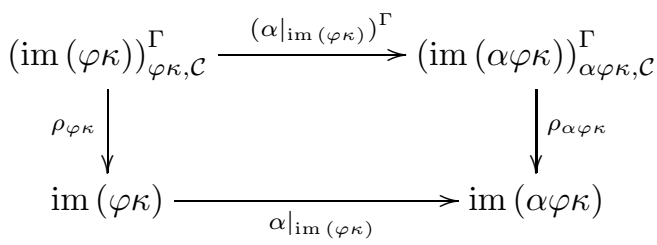

commutes, so the last terms in the above calculations are equal. Therefore we have $\alpha\left(j_{\Gamma}^{\mathcal{C}}(\rho)_{\varphi}(\varphi \kappa)\right)=j_{\Gamma}^{\mathcal{C}}(\rho)_{\psi}\left(\alpha^{\Gamma}(\varphi \kappa)\right)$.

Now we verify $i_{\Gamma}^{\mathcal{C}} j_{\Gamma}^{\mathcal{C}}=\operatorname{id}_{\mathcal{I}_{\Gamma}^{\mathcal{C}}}$. For any $\rho \in \mathcal{I}_{\Gamma}^{\mathcal{C}}$ and homomorphisms $\varphi: \Sigma^{*} \rightarrow M$ in $\mathbf{M}$ and $\iota \in \mathcal{C}\left(\Gamma^{*}, \Sigma^{*}\right)$ we calculate $\left(i_{\Gamma}^{\mathcal{C}} j_{\Gamma}^{\mathcal{C}}(\rho)\right)_{\varphi}(\varphi \iota)=j_{\Gamma}^{\mathcal{C}}(\rho)_{\varphi}(\varphi \iota)=\rho_{\varphi \iota}(\varphi \iota)=$ 
$\rho_{\varphi}(\varphi \iota)$, where the final equality follows from the fact that the inclusion of $\operatorname{im}(\varphi \iota)$ into $M$ is a $\mathcal{C}$-admissible morphism from $\varphi \iota$ to $\varphi$ due to $\iota \in \mathcal{C}\left(\Gamma^{*}, \Sigma^{*}\right)$. Similarly one can also show $j_{\Gamma}^{\mathcal{C}} i_{\Gamma}^{\mathcal{C}}=\operatorname{id}_{\mathcal{I}_{\Gamma}}$. Altogether, the mappings $i_{\Gamma}^{\mathcal{C}}$ and $j_{\Gamma}^{\mathcal{C}}$ are mutually inverse bijections.

To complete the proof it remains to verify that these bijections preserve metric. Because for $\pi, \pi^{\prime} \in \mathcal{I}_{\Gamma}$ the equality $\pi_{\varphi}=\pi_{\varphi}^{\prime}$ immediately implies $i_{\Gamma}^{\mathcal{C}}(\pi)_{\varphi}=i_{\Gamma}^{\mathcal{C}}\left(\pi^{\prime}\right)_{\varphi}$, we obtain $\mathrm{d}\left(i_{\Gamma}^{\mathcal{C}}(\pi), i_{\Gamma}^{\mathcal{C}}\left(\pi^{\prime}\right)\right) \leq \mathrm{d}\left(\pi, \pi^{\prime}\right)$. In order to establish the converse inequality, let $\rho, \rho^{\prime} \in \mathcal{I}_{\Gamma}^{\mathcal{C}}$ and assume $\rho_{\psi}=\rho_{\psi}^{\prime}$ for every $\psi: \Xi^{*} \rightarrow N$ in $\mathbf{M}$ with $|N| \leq n$. Then for any homomorphisms $\varphi: \Sigma^{*} \rightarrow M$ in $\mathbf{M}$ with $|M| \leq n$ and $\kappa \in \mathcal{H}\left(\Gamma^{*}, \Sigma^{*}\right)$ one gets $j_{\Gamma}^{\mathcal{C}}(\rho)_{\varphi}(\varphi \kappa)=\rho_{\varphi \kappa}(\varphi \kappa)=\rho_{\varphi \kappa}^{\prime}(\varphi \kappa)=j_{\Gamma}^{\mathcal{C}}\left(\rho^{\prime}\right)_{\varphi}(\varphi \kappa)$ because $|\operatorname{im}(\varphi \kappa)| \leq n$. Therefore $j_{\Gamma}^{\mathcal{C}}(\rho)_{\varphi}=j_{\Gamma}^{\mathcal{C}}\left(\rho^{\prime}\right)_{\varphi}$ and hence $\mathrm{d}\left(j_{\Gamma}^{\mathcal{C}}(\rho), j_{\Gamma}^{\mathcal{C}}\left(\rho^{\prime}\right)\right) \leq \mathrm{d}\left(\rho, \rho^{\prime}\right)$.

The following corollary is a direct consequence of Proposition 2.4 and the compactness of $\mathcal{I}_{\Gamma}$ provided by Remark 2.3 .

Corollary 2.5. The metric space $\mathcal{I}_{\Gamma}^{\mathcal{C}}$ is compact.

Proposition 2.4 also shows that for any category $\mathcal{C}$ every implicit $\mathcal{C}$-operation can be obtained as the restriction of a unique implicit $\mathcal{H}$-operation to $\mathcal{C}$-admissible $\Gamma$-tuples. Among other things, this allows us to perform substitutions of implicit $\mathcal{C}$-operations in the usual way by the rule $\left(\pi\left(\pi_{1}, \ldots, \pi_{k}\right)\right)_{\varphi}=\pi\left(\left(\pi_{1}\right)_{\varphi}, \ldots,\left(\pi_{k}\right)_{\varphi}\right)$, for $\pi \in \mathcal{I}_{k}$ and $\pi_{1}, \ldots, \pi_{k} \in \mathcal{I}_{\Gamma}^{\mathcal{C}}$, thereby defining a $\Gamma$-ary implicit $\mathcal{C}$-operation $\pi\left(\pi_{1}, \ldots, \pi_{k}\right)$. Notice that for every $\pi \in \mathcal{I}_{k}$ the resulting $k$-ary operation on $\mathcal{I}_{\Gamma}^{\mathcal{C}}$ is uniformly continuous. In particular, this determines a metric monoid structure on $\mathcal{I}_{\Gamma}^{\mathcal{C}}$, where $(\pi \cdot \rho)_{\varphi}(\varphi \iota)=\pi_{\varphi}(\varphi \iota) \cdot \rho_{\varphi}(\varphi \iota)$, and the mappings $i_{\Gamma}^{\mathcal{C}}$ and $j_{\Gamma}^{\mathcal{C}}$ become isomorphisms of the metric monoids $\mathcal{I}_{\Gamma}$ and $\mathcal{I}_{\Gamma}^{\mathcal{C}}$.

\section{Pseudoidentities}

Definition 3.1. For a finite alphabet $\Gamma$, a $\Gamma$-ary $\mathcal{C}$-pseudoidentity is a pair of $\Gamma$-ary implicit $\mathcal{C}$-operations, formally written $\pi \doteq \rho$, where $\pi, \rho \in \mathcal{I}_{\Gamma}^{\mathcal{C}}$. We say that a homomorphism $\varphi: \Sigma^{*} \rightarrow M$ in $\mathbf{M}$ satisfies a $\mathcal{C}$-pseudoidentity $\pi \doteq \rho$ and write $\left.\varphi\right|_{\mathcal{C}} \pi \doteq \rho$ whenever $\pi_{\varphi}=\rho_{\varphi}$. For a set of $\mathcal{C}$-pseudoidentities $T$, the class of all homomorphisms in $\mathbf{M}$ satisfying all $\mathcal{C}$-pseudoidentities in $T$ is $\operatorname{denoted} \operatorname{Mod}_{\mathcal{C}}(T)$.

For any subclass $\mathbf{V}$ of $\mathbf{M}$ and a finite alphabet $\Gamma$, we write

$$
T_{\Gamma}^{\mathbf{V}}=\left\{(\pi, \rho) \in\left(\mathcal{I}_{\Gamma}^{\mathcal{C}}\right)^{2}|\forall \varphi \in \mathbf{V}: \varphi|_{\mathcal{C}} \pi \doteq \rho\right\}
$$

i.e. $T_{\Gamma}^{\mathbf{V}}$ is the set of all $\Gamma$-ary $\mathcal{C}$-pseudoidentities satisfied by all homomorphisms belonging to $\mathbf{V}$. It is clear that $T_{\Gamma}^{\mathbf{V}}$ is a congruence of the monoid $\mathcal{I}_{\Gamma}^{\mathcal{C}}$. We denote by $\nu^{\mathbf{V}}: \mathcal{I}_{\Gamma}^{\mathcal{C}} \rightarrow \mathcal{I}_{\Gamma}^{\mathcal{C}} / T_{\Gamma}^{\mathbf{V}}$ the natural projection homomorphism to the factormonoid. If we define a metric on $\mathcal{I}_{\Gamma}^{\mathcal{C}} / T_{\Gamma}^{\mathbf{V}}$ by the usual formula $\mathrm{d}\left(\pi T_{\Gamma}^{\mathbf{V}}, \rho T_{\Gamma}^{\mathbf{V}}\right)=2^{-r}$, where $r=\min \left\{|M| \mid \exists \varphi \in \mathbf{V}, \varphi: \Sigma^{*} \rightarrow M, \pi_{\varphi} \neq \rho_{\varphi}\right\}$, then $\nu^{\mathbf{V}}$ is easily seen to be continuous and therefore $\mathcal{I}_{\Gamma}^{\mathcal{C}} / T_{\Gamma}^{\mathrm{V}}$ is compact by Corollary 2.5. Finally denote $\nu_{\Gamma}^{\mathbf{V}}=\nu^{\mathbf{v}} i_{\Gamma}^{\mathcal{C}} \nu_{\Gamma}: \Gamma^{*} \rightarrow \mathcal{I}_{\Gamma}^{\mathcal{C}} / T_{\Gamma}^{\mathbf{V}}$ 
Lemma 3.2. Let $\mathbf{V}$ be a subclass of $\mathbf{M}$ and $\varphi: \Sigma^{*} \rightarrow M$ be a homomorphism in $\mathbf{M}$ such that $\varphi \models_{\mathcal{C}} \pi \doteq \rho$ for every $(\pi, \rho) \in T_{\Sigma}^{\mathbf{V}}$. Then there exists a unique continuous homomorphism $\mu_{\mathbf{V}}: \mathcal{I}_{\Sigma}^{\mathcal{C}} / T_{\Sigma}^{\mathbf{V}} \rightarrow M$ satisfying $\mu_{\mathbf{V}} \nu_{\Sigma}^{\mathbf{V}}=\varphi$; it is given by the formula $\mu_{\mathbf{V}}\left(\pi T_{\Sigma}^{\mathbf{V}}\right)=\pi_{\varphi}(\varphi)$.

Proof. Due to Remark 2.3 and Proposition 2.4 there exists a unique continuous homomorphism $\mu_{\mathcal{C}}: \mathcal{I}_{\Sigma}^{\mathcal{C}} \rightarrow M$ such that $\mu_{\mathcal{C}} i_{\Sigma}^{\mathcal{C}} \nu_{\Sigma}=\varphi$ holds, namely $\mu_{\mathcal{C}}=\mu j_{\Sigma}^{\mathcal{C}}$. Then $\mu_{\mathcal{C}}(\pi)=\pi_{\varphi}(\varphi)$ for every $\pi \in \mathcal{I}_{\Sigma}^{\mathcal{C}}$ by the rules for $\mu$ and $j_{\Sigma}^{\mathcal{C}}$ given in Remark 2.3 and Proposition 2.4 respectively. Therefore the assumption of the lemma implies ker $\nu^{\mathbf{V}}=T_{\Sigma}^{\mathbf{V}} \subseteq$ ker $\mu_{\mathcal{C}}$ and so the formula in the statement of the lemma indeed defines a homomorphism $\mu_{\mathbf{V}}: \mathcal{I}_{\Sigma}^{\mathcal{C}} / T_{\Sigma}^{\mathbf{V}} \rightarrow M$ satisfying $\mu_{\mathbf{V}} \nu_{\Sigma}^{\mathbf{V}}=\varphi$. In order to prove that $\mu_{\mathbf{V}}$ is continuous, take any subset $C \subseteq M$ and let us show that $\mu_{\mathbf{V}}^{-1}(C)$ is closed. Since $\mu_{\mathcal{C}}$ is continuous, the set $\mu_{\mathcal{C}}^{-1}(C) \subseteq \mathcal{I}_{\Sigma}^{\mathcal{C}}$ is closed. Because $\mathcal{I}_{\Sigma}^{\mathcal{C}}$ is compact due to Corollary 2.5, this set is also compact. Hence $\mu_{\mathbf{V}}^{-1}(C)=$ $\nu^{\mathbf{V}}\left(\mu_{\mathcal{C}}^{-1}(C)\right)$ is compact and consequently closed as well.

The uniqueness of $\mu_{\mathbf{V}}$ is clear from the uniqueness of $\mu_{\mathcal{C}}$.

Now we are ready to prove that $\mathcal{C}$-pseudovarieties are exactly classes of homomorphisms definable by $\mathcal{C}$-pseudoidentities.

Theorem 3.3. Let $\mathbf{V}$ be a subclass of $\mathbf{M}$. Then $\mathbf{V}$ is a $\mathcal{C}$-pseudovariety if and only if $\mathbf{V}=\operatorname{Mod}_{\mathcal{C}}(T)$ for some set of $\mathcal{C}$-pseudoidentities $T$.

Proof. "£" First we prove that the class $\operatorname{Mod}_{\mathcal{C}}(T)$ is closed under factors. Let a homomorphism $\varphi: \Sigma^{*} \rightarrow M$ in $\mathbf{M}$ satisfy a $\Gamma$-ary $\mathcal{C}$-pseudoidentity $\pi \doteq \rho$ and let $\alpha: M \rightarrow N$ be a surjective homomorphism. Then $\alpha$ is a $\mathcal{C}$-admissible morphism from $\varphi$ to $\alpha \varphi$, having the identity on $\Sigma^{*}$ as a corresponding homomorphism from $\mathcal{C}$. Therefore for every $\iota \in \mathcal{C}\left(\Gamma^{*}, \Sigma^{*}\right)$ we have $\pi_{\alpha \varphi}(\alpha \varphi \iota)=\pi_{\alpha \varphi} \alpha^{\Gamma}(\varphi \iota)=\alpha \pi_{\varphi}(\varphi \iota)=$ $\alpha \rho_{\varphi}(\varphi \iota)=\rho_{\alpha \varphi} \alpha^{\Gamma}(\varphi \iota)=\rho_{\alpha \varphi}(\alpha \varphi \iota)$, which shows that $\alpha \varphi$ satisfies $\pi \doteq \rho$.

If $\varphi: \Sigma^{*} \rightarrow M$ satisfies a $\mathcal{C}$-pseudoidentity $\pi \doteq \rho$ and $f \in \mathcal{C}\left(\Xi^{*}, \Sigma^{*}\right)$, then the inclusion of $\operatorname{im}(\varphi f)$ into $M$ is a $\mathcal{C}$-admissible morphism from $\varphi f$ to $\varphi$ and it is easy to see that $\varphi f \models_{\mathcal{C}} \pi \doteq \rho$. $\operatorname{Hence}_{\operatorname{Mod}_{\mathcal{C}}}(T)$ is closed under substructures.

The situation with products is equally simple because the $\mathcal{C}$-admissibility of the projections from $\left(\varphi_{1}, \ldots, \varphi_{n}\right)$ to $\varphi_{i}$ is testified by the identity homomorphism on $\Sigma^{*}$.

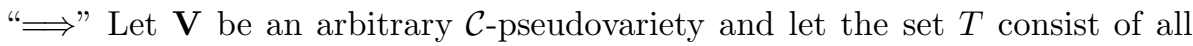
$\mathcal{C}$-pseudoidentities satisfied by all homomorphisms belonging to $\mathbf{V}$. We will prove that $\mathbf{V}=\operatorname{Mod}_{\mathcal{C}}(T)$. The inclusion $\mathbf{V} \subseteq \operatorname{Mod}_{\mathcal{C}}(T)$ is trivial. So take any homomorphism $\varphi: \Sigma^{*} \rightarrow M$ in $\operatorname{Mod}_{\mathcal{C}}(T)$ and consider the unique continuous homomorphism $\mu_{\mathbf{V}}: \mathcal{I}_{\Sigma}^{\mathcal{C}} / T_{\Sigma}^{\mathbf{V}} \rightarrow M$ satisfying $\mu_{\mathbf{V}} \nu_{\Sigma}^{\mathbf{V}}=\varphi$, whose existence is guaranteed by Lemma 3.2. Since $\mu_{\mathbf{V}}$ is a continuous mapping from a compact metric space to a finite discrete space, there exists a positive real number $d_{0}$ such that for every $\pi, \rho \in \mathcal{I}_{\Sigma}^{\mathcal{C}}$ the inequality $\mathrm{d}\left(\pi T_{\Sigma}^{\mathbf{V}}, \rho T_{\Sigma}^{\mathbf{V}}\right) \leq d_{0}$ implies $\mu_{\mathbf{V}}\left(\pi T_{\Sigma}^{\mathbf{V}}\right)=\mu_{\mathbf{V}}\left(\rho T_{\Sigma}^{\mathbf{V}}\right)$. Let $r$ be a positive integer satisfying $2^{-r} \leq d_{0}$ and let $\mathcal{V}$ be a set of representatives of all isomorphism classes of homomorphisms in $\mathbf{V}$ of the form $\psi: \Sigma^{*} \rightarrow N$ where $|N| \leq r$ (i.e. for every homomorphism $\psi: \Sigma^{*} \rightarrow N$ in $\mathbf{V}$ such that $|N| \leq r$ there exists a homomorphism $\psi^{\prime}: \Sigma^{*} \rightarrow N^{\prime}$ in $\mathcal{V}$ and a monoid isomorphism $\alpha: N^{\prime} \rightarrow N$ 
satisfying $\left.\psi=\alpha \psi^{\prime}\right)$. Then the homomorphism

$$
\chi=(\psi)_{\psi \in \mathcal{V}}: \Sigma^{*} \rightarrow \operatorname{im} \chi \subseteq \prod_{\psi \in \mathcal{V}} \operatorname{im} \psi
$$

belongs to $\mathbf{V}$ as well because $\mathcal{V}$ is finite. Therefore Lemma 3.2 can be applied to $\chi$ and we denote by $\bar{\mu}_{\mathbf{V}}$ the unique continuous homomorphism from $\mathcal{I}_{\Sigma}^{\mathcal{C}} / T_{\Sigma}^{\mathbf{V}}$ to im $\chi$ satisfying $\bar{\mu}_{\mathbf{V}} \nu_{\Sigma}^{\mathbf{V}}=\chi$.

In order to verify $\varphi \in \mathbf{V}$ it is enough to show that ker $\bar{\mu}_{\mathbf{V}} \subseteq$ ker $\mu_{\mathbf{V}}$; indeed, then $\mu_{\mathbf{V}}=\alpha \bar{\mu}_{\mathbf{V}}$ for a surjective homomorphism $\alpha: \operatorname{im} \chi \rightarrow M$ and therefore $\varphi=\alpha \chi$ is a factor of $\chi$. Let $\pi, \rho \in \mathcal{I}_{\Sigma}^{\mathcal{C}}$ satisfy $\mu_{\mathbf{V}}\left(\pi T_{\Sigma}^{\mathbf{V}}\right) \neq \mu_{\mathbf{V}}\left(\rho T_{\Sigma}^{\mathbf{V}}\right)$. From the definition of $r$ we know that there exists a homomorphism $\psi: \Xi^{*} \rightarrow N$ in $\mathbf{V}$ with $|N| \leq r$ which does not satisfy the $\mathcal{C}$-pseudoidentity $\pi \doteq \rho$. In other words, there is $\iota \in \mathcal{C}\left(\Sigma^{*}, \Xi^{*}\right)$ such that $\pi_{\psi}(\psi \iota) \neq \rho_{\psi}(\psi \iota)$. Hence the homomorphism $\psi \iota: \Sigma^{*} \rightarrow \operatorname{im}(\psi \iota) \subseteq N$ belongs to $\mathbf{V}$ and because $|\operatorname{im}(\psi \iota)| \leq r$, it is isomorphic to an element of $\mathcal{V}$. If we denote by $\mathrm{p}$ the projection homomorphism from $\operatorname{im} \chi$ to $\operatorname{im}(\psi \iota)$, then we can use the $\mathcal{C}$-admissibility of $\mathrm{p}$ and of the inclusion morphism from $\psi \iota$ to $\psi$ and the formula for $\bar{\mu}_{\mathbf{V}}$ given in Lemma 3.2 to calculate $\mathrm{p}\left(\bar{\mu}_{\mathbf{V}}\left(\pi T_{\Sigma}^{\mathbf{V}}\right)\right)=\mathrm{p} \pi_{\chi}(\chi)=$ $\pi_{\psi \iota} \mathrm{p}^{\Sigma}(\chi)=\pi_{\psi \iota}(\psi \iota)=\pi_{\psi}(\psi \iota)$ and analogously $\mathrm{p}\left(\bar{\mu}_{\mathbf{V}}\left(\rho T_{\Sigma}^{\mathbf{V}}\right)\right)=\rho_{\psi}(\psi \iota)$, which shows $\bar{\mu}_{\mathbf{V}}\left(\pi T_{\Sigma}^{\mathbf{V}}\right) \neq \bar{\mu}_{\mathbf{V}}\left(\rho T_{\Sigma}^{\mathbf{V}}\right)$.

Let us conclude this section by considering relationships between $\mathcal{C}$-pseudoidentities of different arities. First, observe that if the category $\mathcal{C}$ contains all isomorphisms, then for arbitrary $k$-element alphabets $\Gamma$ and $\Delta$, as soon as we fix some bijection between them, the sets $M_{\varphi, \mathcal{C}}^{\Gamma}$ and $M_{\varphi, \mathcal{C}}^{\Delta}$ are naturally isomorphic and therefore there is a one-to-one correspondence between $\Gamma$-ary and $\Delta$-ary implicit $\mathcal{C}$-operations preserving validity of $\mathcal{C}$-pseudoidentities, so it is enough to consider only $k$-ary implicit $\mathcal{C}$-operations for positive integers $k$.

Notice that for a $\Gamma$-ary $\mathcal{H}$-pseudoidentity $\pi \doteq \rho$, to be satisfied as a $\Gamma$-ary $\mathcal{C}$-pseudoidentity means to hold on $\mathcal{C}$-admissible $\Gamma$-tuples. In particular, if $\mathcal{D} \subseteq \mathcal{C}$ then $\varphi \models_{\mathcal{C}} \pi \doteq \rho$ implies $\varphi \models_{\mathcal{D}} \pi \doteq \rho$. For example, if the category $\mathcal{C}$ consists exactly of literal homomorphisms, then a $k$-ary $\mathcal{C}$-pseudoidentity has to be verified on all $k$-tuples of images of letters under $\varphi$; if $\mathcal{C}$ contains precisely injective homomorphisms, a $k$-ary $\mathcal{C}$-pseudoidentity has to be verified on images of all $k$-element codes.

Since $M^{k+1}=M^{k} \times M$, every standard $k$-ary implicit operation $\tau$ determines a $(k+1)$-ary implicit operation $\tau^{\prime}$ by the rule $\tau_{M}^{\prime}\left(e_{1}, \ldots, e_{k}, e_{k+1}\right)=\tau_{M}\left(e_{1}, \ldots, e_{k}\right)$ for $e_{1}, \ldots, e_{k+1} \in M$ and this construction preserves validity of pseudoidentities. But in general there is no such connection between $M_{\varphi, \mathcal{C}}^{k}$ and $M_{\varphi, \mathcal{C}}^{k+1}$. Let us demonstrate this by an example.

Example 3.4. Let the category $\mathcal{C}$ consist of all injective homomorphisms and let $\varphi: \Sigma^{*} \rightarrow M$ in $\mathbf{M}$ satisfy the binary $\mathcal{C}$-pseudoidentity $x y \doteq x$. Then $\varphi$ satisfies also the binary $\mathcal{C}$-pseudoidentity $x^{2} \doteq x$. Indeed, we can consider the injective homomorphisms $f, g:\{x, y\}^{*} \rightarrow\{x, y\}^{*}$ defined by the rules $f(x)=x^{2}, f(y)=y$, $g(x)=x$ and $g(y)=x y$; then for every injective homomorphism $\iota:\{x, y\}^{*} \rightarrow \Sigma^{*}$, 
the compositions $\iota f$ and $\iota g$ are injective too and so we can use the equality of the term operations $x y$ and $x$ on $\varphi \iota f$ and $\varphi \iota g$ to calculate

$$
\varphi \iota\left(x^{2}\right)=\varphi \iota f(x)=\varphi \iota f(x y)=\varphi \iota\left(x^{2} y\right)=\varphi \iota g(x y)=\varphi \iota g(x)=\varphi \iota(x) .
$$

But on the other hand, $\varphi$ does not have to satisfy the unary $\mathcal{C}$-pseudoidentity $x^{2} \doteq x$; just consider $\varphi:\{a\}^{*} \rightarrow \mathbb{Z}_{2}$, where $\mathbb{Z}_{2}$ is a 2-element group. The reason for this is that $\left(\mathbb{Z}_{2}\right)_{\varphi, \mathcal{C}}^{\{x\}} \neq \emptyset$ in spite of $\left(\mathbb{Z}_{2}\right)_{\varphi, \mathcal{C}}^{\{x, y\}}=\emptyset$.

In fact, the equivalence of a given $k$-ary pseudoidentity and the corresponding $(k+1)$-ary pseudoidentity is obtained by considering the inclusion of a $k$-element alphabet into a $(k+1)$-element alphabet and some mapping in the reverse direction identical on these $k$ letters. Therefore, if every homomorphism of free monoids determined by an injective mapping of their underlying alphabets belongs to $\mathcal{C}$, then if a $k$-ary $\mathcal{C}$-pseudoidentity is satisfied by a given homomorphism, it is also satisfied when understood as a $(k+1)$-ary $\mathcal{C}$-pseudoidentity. Conversely, if every homomorphism of free monoids determined by an onto mapping of their underlying alphabets belongs to $\mathcal{C}$, then each $k$-ary $\mathcal{C}$-pseudoidentity is satisfied whenever it is satisfied as a $(k+1)$-ary $\mathcal{C}$-pseudoidentity. Altogether, if $\mathcal{C}$ contains all literal homomorphisms, corresponding pseudoidentities of different arities are equivalent.

\section{EXAMPLE}

For this section, let the category $\mathcal{C}$ consist of all length-multiplying homomorphisms, i.e. such homomorphisms $f: \Sigma^{*} \rightarrow \Xi^{*}$ that there exists a non-negative integer $n$ satisfying $|f(a)|=n$ for all $a \in \Sigma$, where $|f(a)|$ denotes the length of the word $f(a)$. In this case a pseudoidentity is satisfied as a $\mathcal{C}$-pseudoidentity if it holds on tuples of images of words of equal length; notice that for any homomorphism $\varphi: \Sigma^{*} \rightarrow M$ in $\mathbf{M}$ one can effectively decide whether a given $k$-tuple $\left(e_{1}, \ldots, e_{k}\right) \in M^{k}$ is of this form, namely the elements $e_{1}, \ldots, e_{k}$ are images of words of the same length if and only if the regular set of non-negative integers $\bigcap_{l=1}^{k} \#\left(\varphi^{-1}\left(e_{l}\right)\right)$ is not empty, where $\#: \Sigma^{*} \rightarrow \mathbb{N}_{0}$ is the homomorphism mapping each word to its length.

The following construction of $\mathcal{C}$-pseudovarieties was presented in [7].

Definition 4.1. Let $\varphi: \Sigma^{*} \rightarrow M$ be a homomorphism to a finite monoid $M$. The stable subsemigroup of $\varphi$ (written as $\operatorname{Stab} \varphi$ ) is the (unique) subsemigroup $S$ of $M$ of the form $\varphi\left(\Sigma^{m}\right)$ for a positive integer $m$ satisfying $S \cdot S=S$, where $\Sigma^{m}$ is the set of words of length $m$ over $\Sigma$. In other words, $\operatorname{Stab} \varphi$ is the idempotent $(\varphi(\Sigma))^{\omega}$ in the power monoid of $M$, i.e. Stab $\varphi=\varphi\left(\Sigma^{\left(2^{|M|}\right) !}\right)$.

Definition 4.2. For a class $\mathbf{V}$ of finite semigroups, let SV denote the subclass of $\mathbf{M}$ consisting of all homomorphisms whose stable subsemigroups belong to $\mathbf{V}$.

As stated in [7], for any pseudovariety $\mathbf{V}$ of finite semigroups, the class $\mathrm{SV}$ is a $\mathcal{C}$-pseudovariety. In the following we show how to construct a basis of $\mathcal{C}$-pseudoidentities for SV from a basis of pseudoidentities for $\mathbf{V}$. Let us denote for a $k$-ary 
pseudoidentity $\pi \doteq \rho$, where $\pi, \rho \in \mathcal{I}_{k}$, by $\mathrm{S}(\pi \doteq \rho)$ the pseudoidentity

$$
\pi\left(x_{1} y_{1}^{\omega-2} z_{1}, \ldots, x_{k} y_{k}^{\omega-2} z_{k}\right) \doteq \rho\left(x_{1} y_{1}^{\omega-2} z_{1}, \ldots, x_{k} y_{k}^{\omega-2} z_{k}\right)
$$

where $x_{1}, \ldots, x_{k}, y_{1}, \ldots, y_{k}, z_{1}, \ldots, z_{k}$ are new variables and the notation $y^{\omega-2}$ stands for $\lim _{n \rightarrow \infty} y^{n !-2}$.

Proposition 4.3. Let $\varphi: \Sigma^{*} \rightarrow M$ be a homomorphism in $\mathbf{M}$ and $\pi, \rho \in \mathcal{I}_{k}$. Then $\operatorname{Stab} \varphi \models \pi \doteq \rho$ if and only if $\varphi \models_{\mathcal{C}} \mathrm{S}(\pi \doteq \rho)$.

Proof. It is enough to prove the following claim:

Claim 4.4. A $k$-tuple $\left(e_{1}, \ldots, e_{k}\right) \in M^{k}$ of elements of $M$ belongs to $(\operatorname{Stab} \varphi)^{k}$ if and only if there exist words $u_{1}, \ldots, u_{k}, v_{1}, \ldots, v_{k}, w_{1}, \ldots, w_{k} \in \Sigma^{*}$ of equal length such that $e_{l}=\varphi\left(u_{l}\right)\left(\varphi\left(v_{l}\right)\right)^{\omega-2} \varphi\left(w_{l}\right)$ for $l=1, \ldots, k$.

The 'if' part of the claim follows from the fact $\left|u_{l} v_{l}^{n !-2} w_{l}\right|=\left|u_{l}\right| \cdot n$ !, which means that for $n \geq 2^{|M|}$ we have $\varphi\left(u_{l}\right)\left(\varphi\left(v_{l}\right)\right)^{n !-2} \varphi\left(w_{l}\right) \in \operatorname{Stab} \varphi$.

Conversely, let $e \in \operatorname{Stab} \varphi$. Because every element of $\operatorname{Stab} \varphi$ can be written as a product of two elements of $\operatorname{Stab} \varphi$, we have $e=f_{1} \cdots f_{|M|+2}$ for some $f_{1}, \ldots, f_{|M|+2} \in \operatorname{Stab} \varphi$. Hence there exist $1 \leq i<j \leq|M|+1$ such that $f_{1} \cdots f_{i}=f_{1} \cdots f_{j}$. Consequently

$$
e=\left(f_{1} \cdots f_{i}\right)\left(f_{i+1} \cdots f_{j}\right)^{\omega-2}\left(f_{j+1} \cdots f_{|M|+2}\right)
$$

and therefore $e$ is of the desired form for some words of length $\left(2^{|M|}\right)$ ! since $\left(f_{1} \cdots f_{i}\right),\left(f_{i+1} \cdots f_{j}\right),\left(f_{j+1} \cdots f_{|M|+2}\right) \in \operatorname{Stab} \varphi$.

Corollary 4.5. If $\mathbf{V}=\operatorname{Mod}\left(\pi_{i} \doteq \rho_{i} \mid i \in I\right)$ is a pseudovariety of finite semigroups, then $\mathrm{SV}=\operatorname{Mod}_{\mathcal{C}}\left(\mathrm{S}\left(\pi_{i} \doteq \rho_{i}\right) \mid i \in I\right)$.

As observed in [7], classes of homomorphisms corresponding to certain classes of regular languages defined by means of boolean circuits are in fact $\mathcal{C}$-pseudovarieties of the form of Definition 4.2. For instance in [2] it was proved that the regular languages in $A C^{0}$ (i.e. recognized by constant-depth unbounded fan-in circuits of polynomial size) are just those languages whose syntactic homomorphisms have aperiodic stable subsemigroups. Due to the previous result, these languages correspond to the $\mathcal{C}$-pseudovariety $\operatorname{Mod}_{\mathcal{C}}\left(\left(x y^{\omega-2} z\right)^{\omega} \doteq\left(x y^{\omega-2} z\right)^{\omega+1}\right)$.

Acknowledgements. I am grateful to Ondřej Klíma and Libor Polák for their useful comments and suggestions.

\section{REFERENCES}

[1] J. Almeida, Finite Semigroups and Universal Algebra. World Scientific, Singapore (1995).

[2] D. Mix Barrington, K. Compton, H. Straubing and D. Thérien, Regular languages in $N C^{1}$. J. Comput. System Sci. 44 (1992) 478-499.

[3] S. Eilenberg, Automata, Languages and Machines. vol. B, Academic Press, New York (1976). 
[4] J.E. Pin, A variety theorem without complementation. Russian Math. (Iz. VUZ) 39 (1995) $74-83$.

[5] J. Reiterman, The Birkhoff theorem for finite algebras. Algebra Universalis 14 (1982) 1-10.

[6] M.P. Schützenberger, On finite monoids having only trivial subgroups. Inform. and Control 8 (1965) 190-194

[7] H. Straubing, On the logical description of regular languages. in Proc. 5th Latin American Sympos. on Theoretical Informatics (LATIN 2002), edited by S. Rajsbaum, Lecture Notes in Comput. Sci., vol. 2286, Springer, Berlin (2002) 528-538.

Communicated by Z. Ésik.

Received December 21, 2002. Accepted September 3, 2003. 